\title{
Vertical and Kink Mode Stability Calculations for Current Carrying Quasiaxial Stellarators
}

\author{
M. H. Redi ${ }^{1}$, C. Nuehrenberg ${ }^{2}$, W. A. Cooper ${ }^{3}$, G-Y. Fu ${ }^{1}$, C. Kessel ${ }^{1}$, \\ L.P.Ku ${ }^{1}$, D. A. Monticello', A. Reiman ${ }^{1}$, M. C. Zarnstorff ${ }^{1}$ \\ ${ }^{1}$ Princeton Plasma Physics Laboratory, Princeton University, Princeton, NJ 08543 \\ ${ }^{2} I P P$, Greifswald, Germany \\ ${ }^{3}$ CRPP-PPB, Lausanne, Switzerland
}

\section{Introduction}

Vertical and kink mode stability are essential for strongly shaped tokamaks, to preclude disruptive plasma termination. Similarly, the design of stellarators with significant current, such as quasiaxial stellarators (QAS) must be carefully examined to ensure adequate vertical and kink stability. The CAS3D [1] and TERPSICHORE [2] MHD stability code packages are being used to evaluate the stability of QAS configurations. CAS3D has previously been applied to predict stability of the W7-X stellarator, under construction at IPP, Greifswald, Germany. As part of a multifaceted effort to develop an interesting compact quasiaxial stellarator configuration for a modest sized experiment at PPPL, these two code packages are being used for three dimensional calculations of internal and external MHD stability, including extensive benchmarking of MHD stability for axisymmetric and nonaxisymmetric cases.

\section{Global MHD Stability Code Packages: CAS3D and TERPSICHORE}

CAS3D is a code package for the analysis of the stability of three dimensional magnetohydrodynamic equilibria which makes use of finite element Fourier decomposition in Boozer coordinates. From the several versions of this code package, CAS3D2vac is used here for calculations of the kink mode and the periodicity-preserving mode (known as the axisymmetric or vertical mode in tokamaks). Version CAS3D2MN, with a phase factor transform, was used for the fixed boundary two field period QAS benchmarking calculations. TERPSICHORE has many similar features and has recently been extended to calculations of the periodicity-preserving mode. It has been written for efficient computation and is being used in configuration optimization of the compact stellarator design.

\section{Calculations for Internal Mode Stability of a Quasiaxially Symmetric Stellarator}

During 1997-1998 we carried out benchmarking studies with CAS3D and TERPSICHORE which examined Mercier unstable modes for a compact two field period QAS with $20 \%$ external transform. These modes, which are resonant modes of the fixed boundary equilibrium, exhibit relatively delocalized, but not globally unstable, displacements (see Fig. 1). The radial shape and relative ordering of the largest amplitude Fourier components of unstable modes have been compared for coarse (49 surfaces) and fine grid (129 flux surfaces), as well as at values of $\beta=6.7,6.5,6.4,6.3,6.0,5.0 \%$ with CAS3D and TERPSICHORE. Good agreement was found in every case. For example as shown in Fig. 1, at $6.7 \%$ beta and 48 flux surfaces CAS3D identified the five largest amplitude (m,n) components of the most unstable mode as $(11,-5)(9,-3)(16,-7)(14,-5)(6,-3)$ while TERPSICHORE identified them as $(11,5)(6,3)(9,3)(16,7)(14,5)$. The opposite signs in the toroidal Fourier indices originate from the different notations used in the Fourier representations. CAS3D uses $\cos (2 \pi(m \theta+n \phi))$, while TERPSICHORE uses $\cos (2 \pi(m \theta-$ $\mathrm{n} \phi)$ ). The poloidal and toroidal grids were not matched and are expected to account for the differences in ordering. Results from the two stability code packages for the marginal beta in fixed boundary equilibria (Fig. 2) are also in good agreement for this two field period QAS. 


\section{Calculations of Stability of the Kink and Periodicity-preserving Modes for Axisymmetric and Quasiaxisymmetric Configurations}

The NCSX stellarator configuration [3] has been optimized for the stability of ballooning, kink and periodicity-preserving modes. TERPSICHORE has been used to show that high edge magnetic shear and appropriate boundary shaping can stabilize the kink mode in high bootstrap current QAS [4]. CAS3D has confirmed TERPSICHORE calculations [5] of stability for the kink and periodicity-preserving modes for the three field period, 50\% external transform stellarator and extended them, finding stability even without a conducting wall.

TERPSICHORE has been benchmarked against an analytical solution for the critical wall position for vertical stability in a large aspect ratio tokamak with good agreement. In addition the vertical stability has been calculated for a series of zero beta equilibria interpolated between the present design and a tokamak equilibrium. The tokamak (terms with $n=0$ ) is unstable. As nonaxisymmetric terms are added, the growth rate decreases, reaching zero about halfway to the present design. The periodicity-preserving mode stability thus appears robust. Calculations at zero beta were carried out because of difficulty converging the high beta, low transform tokamak equilibrium. Finite beta is found to stabilize this mode for the nonaxisymmetric equilibrium with the wall at $r=4.5 \mathrm{a}$. (See Ref. 5).

CAS3D has calculated stability in NCSX for the kink $(\mathrm{N}=1)$ and the periodicitypreserving $(\mathrm{N}=0)$ modes, with a conducting wall at infinite distance from the plasma boundary. Figure 3 shows an analysis of local stability criteria for an equlibrium with a locally reduced pressure gradient. Pressure and iota profiles are shown, as well as the criteria for the local Mercier resonance instabilities. Most of these resonances are narrow, so that in their vicinity the Mercier term in the global stability can recover. However at the outside edge of the plasma, for $\mathrm{s}>0.6$, broad resonances occur and the Mercier term is unstable. Clearly the singular resonant currents are unphysical and improved physics models are needed to handle them properly. One approach to this, flattening of the pressure profile at the resonances. does reduce their width and stabilizes them. However, with a locally reduced pressure gradient, including the Mercier resonances in the global stability calculations leads to predictions of instability for both the kink and periodicity-preserving modes (Fig. 4).

CAS3D benchmarking calculations in two dimensions for the stability of the external kink for ARIES are in good agreement with predictions of TERPSICHORE and the PEST [6] codes. Table I shows the PEST comparisions with CAS3D and TERPSICHORE for kink stability for this axisymmetric equilibrium. The CAS3D vertical stability calculations of the axisymmetric case show instability for elongated tokamaks, and stability for circular tokamaks (Table II). The most unstable harmonics of the vertical instability for the ARIES axisymmetric tokamak at $7.5 \%$ beta for $m>1$ are suppressed at lower beta (2.1\%).

There remain questions concerning the best current and pressure profiles to use for design optimizaton. Most stellarator density profiles are broader than the ARIES profiles assumed in initial studies for NCSX. Also the current profile was assumed to be similar to a low collisionality reactor bootstrap current profile, to enable rapid reactor performance scaling. Work is ongoing to address flexible performance in a modest-sized experiment.

\section{Conclusion}

Optimized configurations have been identified for the proposed NCSX experiment which are stable to ballooning, kink and the periodicity-preserving modes and have acceptable confinement. Neoclassical particle transport is being studied and optimized for good confinement [7]. The three dimensional global ideal MHD stability code packages CAS3D and TERPSICHORE are found in agreement in numerous benchmarking studies and both predict global stability for the NCSX design point configuration. CAS3D has extended TERPSICHORE calculations for NCSX showing stability of the kink and periodicitypreserving modes for this stellarator even without a conducting wall. There is still a need for comprehensive comparisons of detailed measurements of MHD instabilities to results of global stability calculations for both rippled tokamak and stellarator experiments. 
* Supported by the US Dept. of Energy Contract DE-AC02-76CH03073.

[1] C. Neuhrenberg, Phys. Plas. 3, 2401 (1996). ]C, Schwab Phys.Fluids B 5, 3195 (1993).

[2] W. A. Cooper, et al., Phys. Plas. 3, 275, (1996).

[3] R. C. Grimm, et al., Meth. in Comput. Phys. 16, 273 (1975).

[4] A. H. Reiman, "Physics Design of a High Beta Quasi-axisymmetric Stellarator", this conference, paper TL18.

[5] G. Y. Fu, et al, IAEA-CN-69/THP1/07, 17 $7^{\text {th }}$ IAEA Fusion Energy Conf., Yokahama, 1998.

[6] G. Y. Fu, et al, "Ideal MHD Stability in High Beta Current-Carrying Quasi-axisymmetric Stellarators", in preparation.

[7] M. H. Redi, et al, Phys. Plas. In press (Sept., 1999).

Table I Kink Stability

$\beta$ CAS3D PEST TERPSICHORE PEST

(\%) (wall at infinity) (wall at $\mathrm{r}=2 \mathrm{a}$ )

7.5 unstable unstable unstable unstable

3.6 unstable unstable unstable unstable

2.5 unstable unstable stable stable

2.1 stable stable stable stable
Table II Vertical Stability

$\beta \quad$ eigenvalue

elongated

$7.5 \% \quad-0.041$

$3.5-0.076$

$2.5-0.089$

$2.1 \quad-0.094$

circular

$0.7 \quad-3.10^{-12}$ 

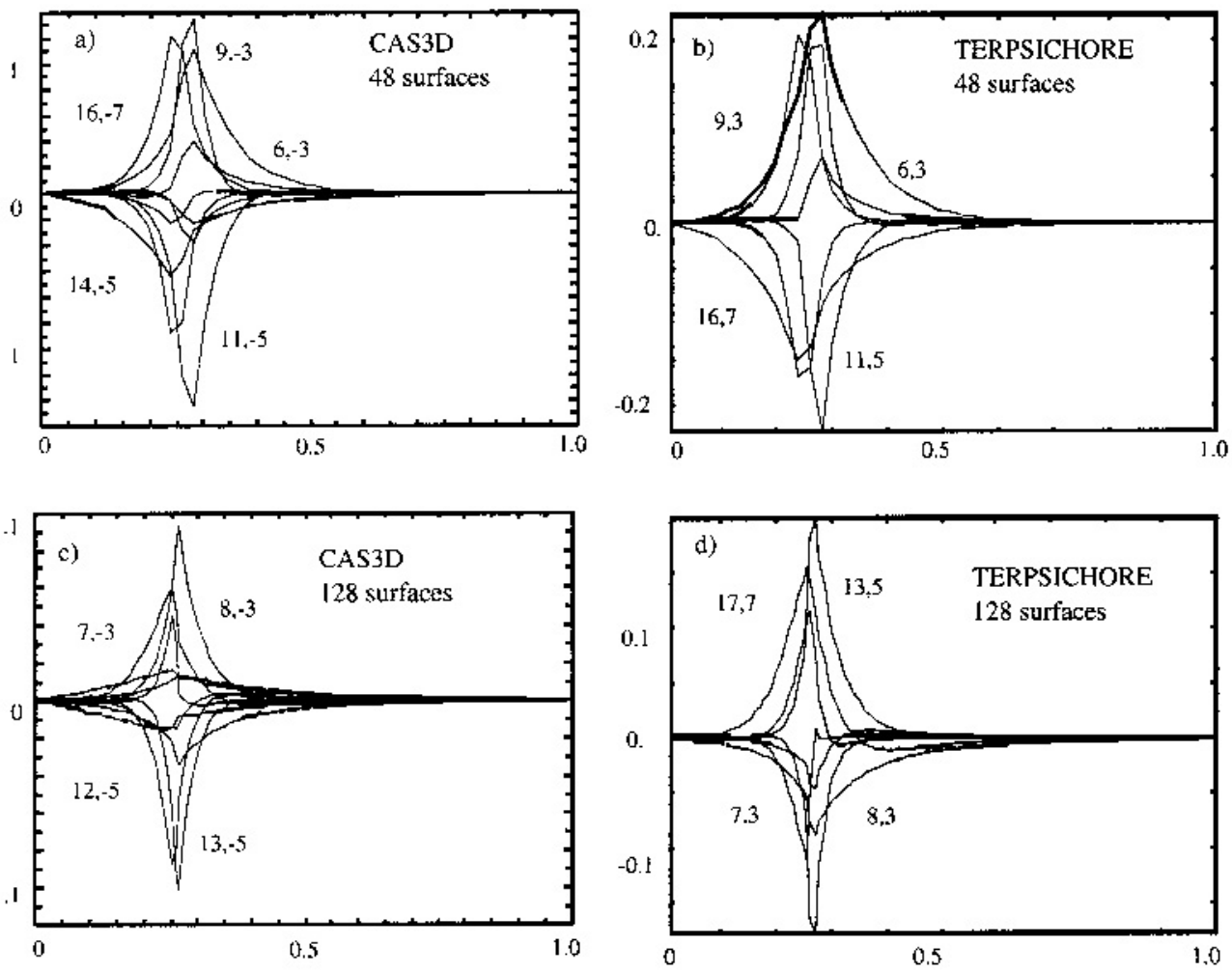

Fig. I Largest Fourier components of most unstable mode calculated for QAS2_20 at 6.7\% beta, for 48 flux surfaces $(a) \lambda=-1.3$ ) and 129 flux surfaces $(c) \lambda=-0.35$ ) by CAS3D, and for 48 flux surfaces, $(b) \lambda=-0.02$ and for 129 flux surfaces $(d) \lambda=-0.007$ by TERPSICHORE . 


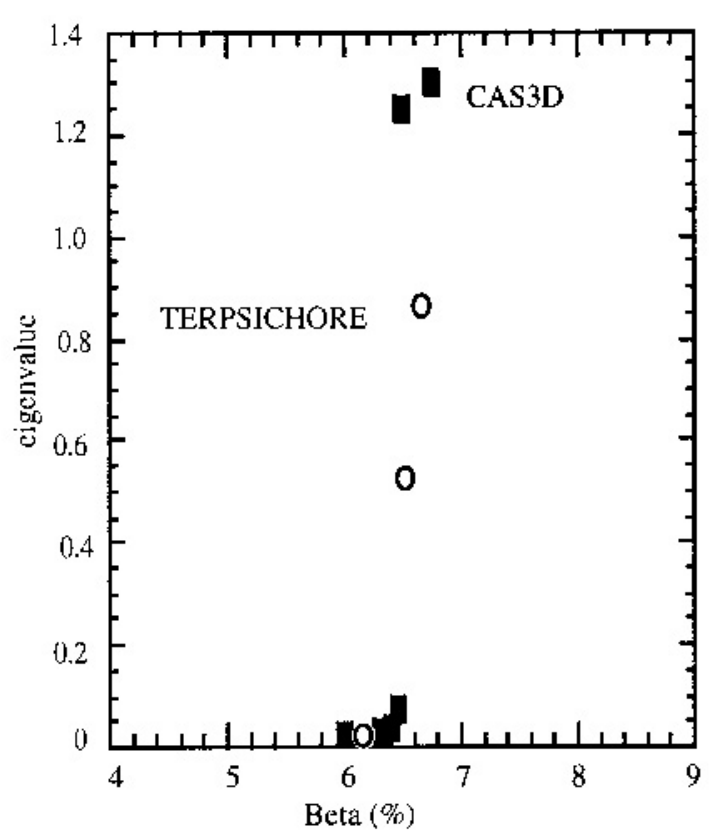

Fig. 2. Marginal beta point for QAS2_..20 found by CAS3D (solid squares) and TERPSICHORE (open circles) is $6.5 \%$ beta. TERPSICHORE eigenvalues are multiplied by 20 ; the two codes are normallized differently.

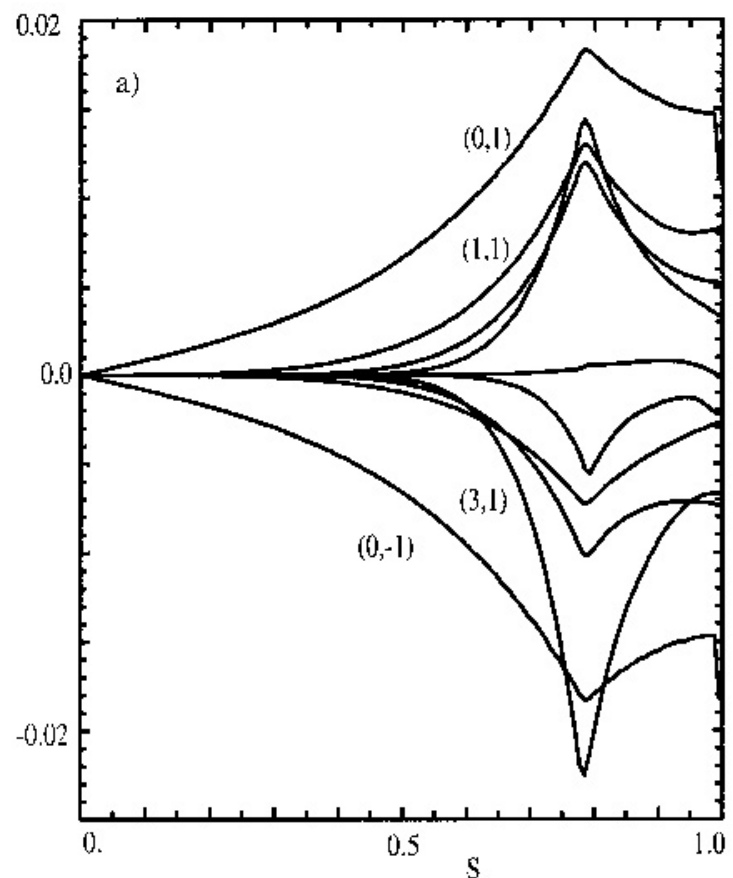

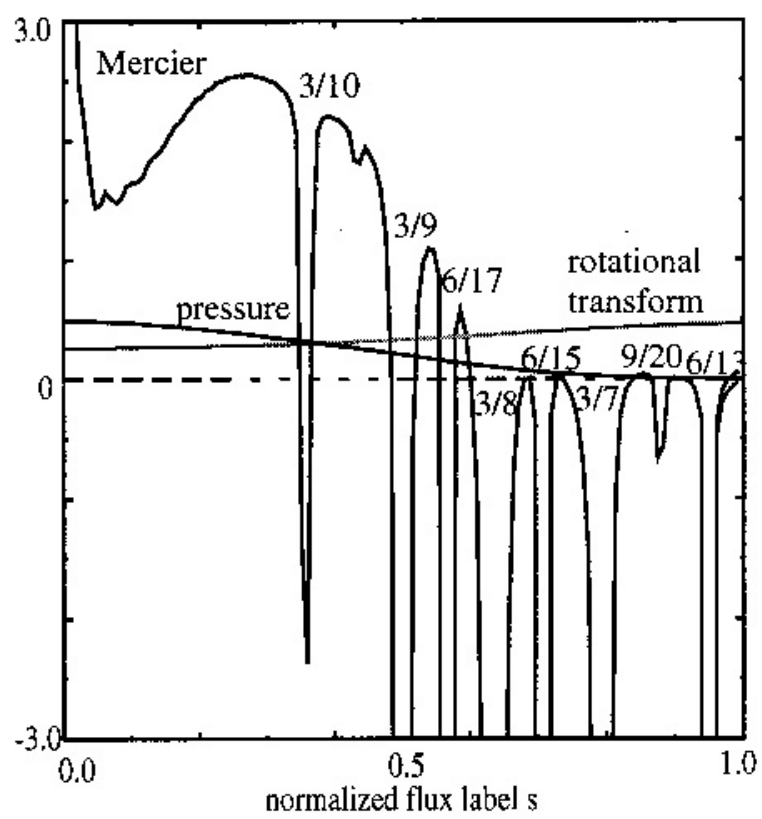

Fig. 3 Local stability profiles for QAS3_C82, showing pressure $(10 \mathrm{x})$, rotational transform, and Mercier stability (positive for stability).

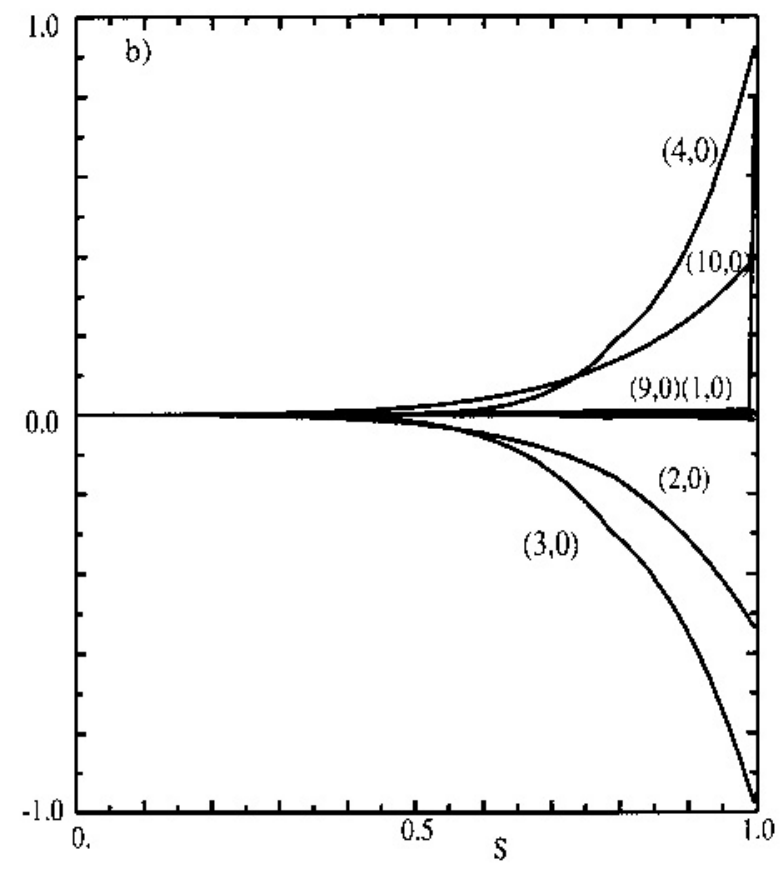

Fig. 4. Largest Fourier components of a) the $\mathrm{N}=1$, kink mode instability and b) the $\mathrm{N}=0$, vertical or axisymmetric mode instability for QAS3_C82 as calculated from CAS3D if natural resonances, Mercier instabilities, are not eliminated. Simulation parameters: 25 modes, 128 flux surfaces. 